\title{
IMPROVEMENT OF FABRIC DETECTION ALGORITHM BASED ON SOBEL OPERATOR
}

\section{Computer Science}

Zhang Chao*

Yang Lianhe
School of computer science and technology, TianGong University, Tianjin, 300387, China. * Corresponding Author

School of computer science and technology, TianGong University, Tianjin, 300387, China.

\section{ABSTRACT}

The traditional Sobel operator has incomplete edge detection, and improper selection threshold causes edge judgment error. In this paper, nonmaximum suppression combined with adaptive threshold selection is proposed for fabric defect detection. This method uses bilateral filtering for image preprocessing to eliminate the influence of noise and illumination imbalance on the image. Increase by 45 per cent。and 135 。 gradient calculation in two directions, using non-maximum suppression algorithm to refine the image edge, and reduce the misjudgment of edge points by adaptive threshold selection.

\section{KEYWORDS}

sobel operator,adaptive threshold,defect detection

\section{INTRODUCTION}

China is a textile country, the quality of textile products determines the company's income, in weaving mass production, because of raw materials, machinery, manual operation and other reasons, defects, defects of many kinds of [1]. Nowadays, most factories choose to carry out defect detection manually. It takes 6 to 8 minutes for a successful worker to test. The factory environment is very bad, the damage to human body is very serious, and people have limits. With the increase of working hours, the error rate will gradually increase, so the cost will be higher. Automatic defect detection technology research, textile industry has great significance [2]. In order to solve this problem, many experts carry out in-depth research and put forward many methods. Now the mainstream methods are model analysis, statistics, spectrum [2], Canny edge detection algorithm and so on. The method of model analysis is to model the texture information of the fabric itself and form a mathematical model, but the calculation is large and it is not suitable to identify the smaller defects. Statistical method: through the statistical gray value, through the feature vector accurate location of defects. This method is simple, easy to operate, but the requirements for pictures are relatively large, easy to be affected by the external environment. Spectrum method: the unique texture in the fabric is as periodic as the spectrum. In the processing of fabric texture, Fourier transform transforms the two-dimensional gray distribution of the image into the spectrum of the corresponding two-dimensional space domain, and analyzes the texture in the frequency domain. This method is only suitable for global, and it is not very good for dealing with local defects. Wang Chunyan [3] et al. proposed fabric edge detection based on Prewitt operator to improve the reliability of Prewitt operator. Hu Keman et al proposed an adaptive Gauss filter parameters and threshold Canny edge detection algorithm to improve the adaptability of the method and improve the ability of edge detection.Because the texture structure of the defective white textile is different from that of the normal, the density gradient will appear in the edge part of these defects and the normal part. Because the edge detection algorithm is simple and efficient, the edge detection algorithm is adopted. The commonly used edge detection algorithms are Canny edge detection, Sobel operator, Scharr operator and so on. Python is used to program each algorithm.

Sobel operator combines Gauss smoothing and differential derivation operation to find edges by local difference. In the traditional edge detection algorithm, edge detection and smoothing can not achieve the best at the same time. For this problem, this paper proposes an improved method based on Sobel operator :(1) increase 45. and 135. The template direction is used to improve the edge structure of the image .(2) the non-maximum suppression method is used to refine the detected edge structure .(3) the adaptive threshold is used to remove some pseudo edges.

\section{Classic Sobel Edge Detection}

Classical Sobel detection algorithm is a local difference for edge detection. The traditional template is shown in figure 1 to 2 :

\begin{tabular}{|l|l|l|}
\hline-1 & 0 & 1 \\
\hline-2 & 0 & 2 \\
\hline-1 & 0 & 1 \\
\hline
\end{tabular}

Figure1 x direction matrix

Figure $2 \mathrm{y}$ direction matrix The convolution formula is as follows: $G_{i}=\sum_{m=-1}^{1} \sum_{n=-1}^{1} f(x+m, k+n) M_{i}$

(1) $\mathrm{G}$ is the gradient value after convolution and the i value is 0 。,90。 Two edge directions, M Sobel corresponding operator template. According to the above situation, the traditional Sobel operator can only be solved in X,Y two directions, and it is easy to ignore the edges of other directions. Moreover, the selection of threshold is obtained according to manual experience, and the threshold is too high, which leads to partial edge loss, low threshold and edge redundancy.

\section{An Improved Sobel Operator Defect Detection Algorithm}

On the basis of the classical algorithm, we first perform Gaussian filtering, adding to the previous two directions 45 。 and 135。.Two directions calculate gradient value, non-maximum suppression, adaptive threshold selection, the flow chart is as follows:

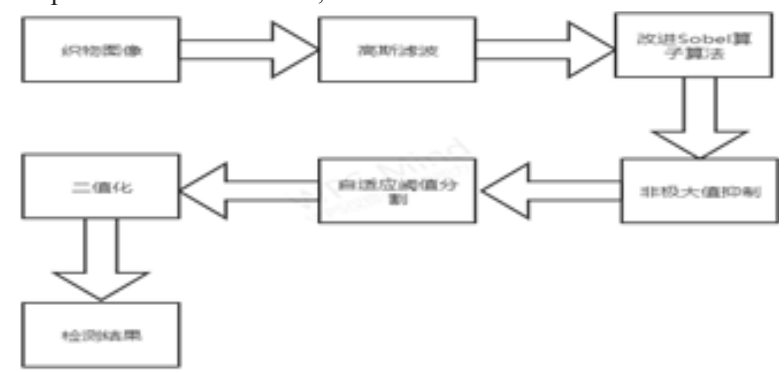

Figure 3 flow chart

\section{Gaussian Filters}

Gauss filter is a method of image preprocessing and a linear smoothing filter[5]The principle is to use a convolution template to move each pixel on the image, and the weighted average of the pixels in the field is the gray value of the pixel as follows:

$F(x, y)=\frac{1}{2 \pi \sigma^{2}} e^{\frac{-x^{2}-y^{2}}{2 \sigma^{2}}}$

$\sigma(x, y)$ is the coordinate of the pixel point and is the standard deviation, which determines the degree of denoising smoothness.

\section{Improved Sobel Operator Algorithm}

Although the traditional Sobel operator adopts the method of local difference, it can only detect the vertical and horizontal directions, 
which leads to the loss of edge information, and the improved algorithm increases by 45 。 and 315 。 Two directions to make the edge structure more complete. The additional operator templates are

\begin{tabular}{|c|c|c|}
\hline-2 & -1 & 0 \\
\hline-1 & 0 & 1 \\
\hline 0 & 1 & 2 \\
\hline
\end{tabular}

Figure $345^{\circ}$ direction matrix

\begin{tabular}{|l|l|l|}
\hline 0 & -1 & -2 \\
\hline 1 & 0 & -1 \\
\hline 2 & -1 & 0 \\
\hline
\end{tabular}

Figure $4135^{\circ}$ direction matrix

Combined with the two operator modules in Fig .2, the four operator modules are calculated by formula 1 . The improved gradient amplitude is $\mathrm{f}(\mathrm{x}, \mathrm{y})$ :

$$
f(x, y)=\max \left\{G_{0}, G_{45}, G_{180}, G_{315}\right\}
$$

\section{Maximum suppression of gradient amplitude}

The effect of maximum inhibition is to refine the edge[6]When the gradient direction is 0 。 When the edge of the image is northwest, the current pixel is compared with the left and right sides. If the gradient amplitude of the current pixel is the largest, it is retained, otherwise it is eliminated. When the gradient direction is 90 。 When the edge of the image is east-west, the current pixel is compared with the gradient value of the upper and lower pixels. If the current pixel gradient value is the largest, it is retained, otherwise it is eliminated. When the gradient direction is 45 。 The edge of the image is east, south and northwest. The current pixel[7] is compared with the gradient in the upper right corner and the lower left corner. If the gradient amplitude of the current pixel is the largest, it is retained, otherwise it is eliminated. When the gradient direction is 315 。 The edge of the image is southwest-northeast[8]. The current pixel is compared with the gradient value of the upper left and lower right corner pixels. If the gradient amplitude of the current pixel is the largest, it is retained, otherwise it is eliminated.

\section{Adaptive threshold}

As a result of I, Gaussian smoothing, the formula of

adaptive threshold matrix is: $f_{\text {smooth }}(I)$

Thresh $=(1-$ ratio $) * f_{\text {smooth }}(I)$

According to many experiments, the ratio value is 0.15 . Threshold. The principles of adaptive threshold segmentation are:

$o(r, c)=\left\{\begin{array}{l}255, I(r, c)>\operatorname{Thresh}(r, c) \\ 0, I(r, c) \geq \operatorname{Thresh}(r, c)\end{array}\right.$

Experimental Results and Analysis

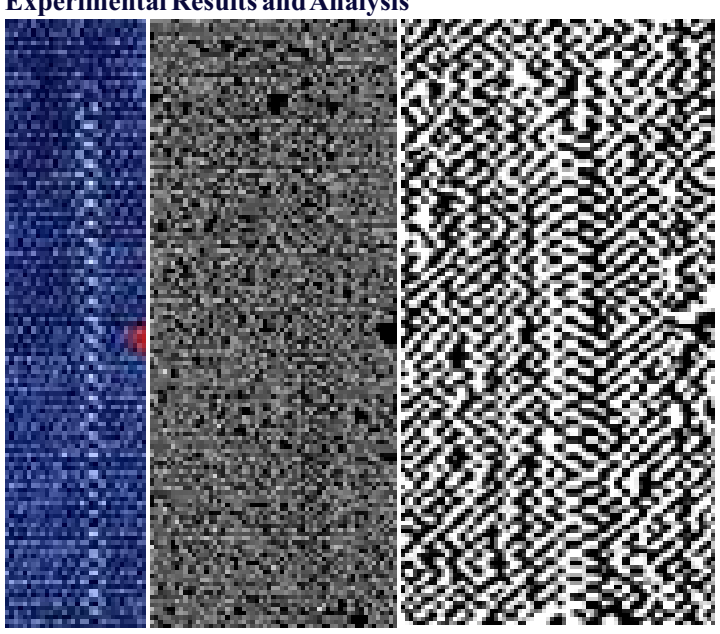

Original Canny operator Sobel operator

Figure 5 experimental result

As shown in figure 5, the improved Sobel operator has advantages in the commonly used edge detection operators, and can clearly find out the location of defects.

\section{CONCLUSION}

Based on the classical Sobel operator, two-direction templates are added to combine non-maximum suppression and adaptive threshold for fabric detection. compared with other methods, the edge detection method is simple to operate and efficient. Compared with the traditional Sobel operator[9], the improved method solves the edge misjudgment caused by rough edge detection and artificial selection threshold to a great extent, and realizes the intelligence of the edge and has certain adaptability. This method is put into practical application, considering some daily constraints, it needs further study[10]

\section{REFERENCES}

[1] GUAN S ,SHI H.Fabric defect detection based on the saliency map construction of target-driven feature[J]. The Journal of The Textile Institute,201809(9): $1133-11142$.

[2] Zhang Xingye. A Study on Key Technologies of Automatic Detecting System for Fabric Defects [D].]1 Jiangnan University ,2012

[3] Liu Haijun, Shan Weifeng, Han Ying, Li Zhong. An Algorithm for Detecting Textile Defect Based on Gradient Histogram Wordbag [J].] and Wofang Technology 2019,47(03):71-76

[4] Wang Chunyan, Shen Danfeng, Yang Guozhong, Zhang Xuxiang. Modified algorithm for fabric defect detection using Prewitt operator [J].]; and Progress in Textile Technology 2020(03):42-46.

[5] Hu Keman, Luo Shaolong, Hu Haiyan. Modified algorithm for fabric defect detection using Canny operator [J].]; and Journal of Textiles ,2019,40(01):153-158

[6] Li Jian, Ding Xiaoqi, Chen Guang, Sun Jian, Jiang Nan. Blade Image Denoise Method [J].] Based on Improved Gaussian Filter Journal of Southern Agriculture ,2019,50(06):1385-1391.

[7] Xia Yongquan, Huang Haipeng, Wang Bing. A method for detecting crop disease spots based on improved non-maximum suppression [J].]; and Journal of Light Industry 2017,32(01):97-102.

[8] Deng Ailin. Zhu Yangyong. Collaborative Filtering Recommendation Algorithm Based on Project Scoring Prediction [J]. Journal of Software, 2003, 14(9): 1621-1628.

[9] Zheng Yuping, Hu Minjie, Yang Honghe, et al. Research on collaborative filtering algorithm based on rough set [J/OL]. Journal of Shandong University (Science Edition), 2019, 54(1):1-10.

[10] LIU H, HU Z, MIAN A, et al. A new user similarity model to improve the accuracy of collaborative filtering [J]. Knowledge-Based Systems, 2014, 56(3):156-166. 